\title{
PROSPECTS FOR A CENTRAL THEORY OF PARTIAL DIFFERENTIAL EQUATIONS
}

\author{
J. W. NEUBERGER
}

\section{INTRODUCTION}

Three episodes in the history of equation solving are: Finding roots of polynomials, solution of ordinary differential equations, solution of partial differential equations. The first two episodes went through a number of phases before reaching a rather satisfactory state. That the third episode might develop similarly is the topic of this note.

\section{Roots of Polynomials}

The quadratic formula for finding roots to second order polynomials has been known since antiquity. Cardan's formulae for finding roots to third and fourth degree polynomials were given in the 1400's. Galois demonstrated the impossibility of extending such formulae to higher order polynomials. Nevertheless, roots of polynomials eventually became well understood. Key to this understanding is the matter of existence of roots (fundamental theorem of algebra) and the practical computation of roots numerically. A result from [12] illustrates a modern point of view on this subject.

Suppose $p$ is a non constant complex polynomial. Call $z: R \rightarrow C$ a trajectory of $p$ if $z$ is continuous, has domain all of $R$ and

$$
p(z)^{\prime}=-p(z) .
$$

For a trajectory $z$ of $p$ and $s \in R$, if $p^{\prime}(z(s))=0$ and $p(z(s)) \neq 0$ then

$$
\{z(t): t \leq s\}
$$

is called a half-trajectory of $p$. Denote by $M$ the union of all half-trajectories of $p$.

\section{Theorem 1.}

- Every member of $C$ belongs to some trajectory of $p$.

- If $z$ is a trajectory of $p$, then $u=\lim _{t \rightarrow \infty} z(t)$ exists and $p(u)=0$.

- Each component of the complement of $M$ contains exactly one root of $p$.

A plot of the Newton vector field for $p$ visually picks out good approximations to roots of $p$ since they are the points to which trajectories are converging. Once a point close to a root is identified, ordinary Newton's method can be used to calculate that root with great efficiency. Members of $C$ which terminate half-trajectories are hyperbolic points, more specifically points to which at least two half-trajectories converge and at least two trajectories leave. These can also be identified visually from the Newton vector field plot for $\mathrm{p}$.

Date: 2 January 2005. 


\section{Ordinary Differential Equations}

Some centuries of effort were spent in finding 'closed form' solutions to systems of ordinary differential equations (ODE). Sophus Lie's quest was to find integrating factors for systems of ODE. Lie found many interesting things but he didn't provide the start of a central point of view for ODE. That came with the arrival of existence and uniqueness results. Such results give something about which to establish qualitative properties and for which to calculate numerical approximations. The following is a representative result:

Theorem 2. Suppose $c<d, W$ is an open subset of a Banach space $X$ and $f$ is a $C^{1}$ function from $(c, d) \times W$ to $X$. If $(b, w) \in(c, d) \times W$, there is an open interval $S$ containing $b$ on which there is a unique function $u$ satisfying

$$
u(b)=w, u^{\prime}(t)=f(t, u(t)), t \in S .
$$

This result and its many generalizations give a point of departure for studying a vast variety of problems in ODE.

\section{Steepest Descent in Hilbert Space}

For a central point of view on PDE we look to variational settings. We will see that there are two possibilities for recasting a system of differential equations as a variational problem. Books by Evans [3], Zeidler [20], Struwe [18], to name just three, contain vast amounts of information on variational principles that relate to differential equations. The present article is not a place for an authoritative summary of mainstream work on variational problems in PDE, but we will make a some comparisons. A reader may judge whether or not a starting place for gaining a central point of view on PDE is presented here.

Our development is centered on descent methods which are essentially constructive and as such have a natural numerical counterpart. Our setting is broad enough to allow for a wide variety of supplemental conditions - more general than just 'boundary conditions'. Our descent methods rely upon a construction of a class of gradients of real-valued functions. We have called our gradients 'Sobolev gradients' in recognition of the fact that Sobolev spaces generally form the domains of functionals of interest here. To tell a simpler story we deal mainly with Hilbert spaces.

The plan of this note is as follows. The present section gives some results on continuous steepest descent using Sobolev gradients and the role played by Łojasiewicz inequalities (gradient inequalities). Section 5 contains a quick review of Sobolev spaces and Von Neumann's orthogonal projection onto the graph of a closed densely defined linear operator. Section 6 gives two ways in which Sobolev gradients are used in differential equations: in least-squares formulations and in direct minimization of 'energy' functionals. Section 7 contains two approaches to the matter of boundary conditions: by means of foliations and by nonlinear constrained optimization. Section 8 contains a related Nash-Moser inverse function theorem which avoids 'loss of derivatives' by means of a version of continuous Newton's method. In Section 9 applications are illustrated by means of results for the full GinzburgLandau energy functional for superconductivity. 
To begin, suppose that $H$ is a real Hilbert space and $\phi$ is a real-valued $C^{1}$ function on $\mathrm{H}$. A critical point of $\phi$ is an element $u$ of $H$ so that

$$
\phi^{\prime}(u) h=0, h \in H .
$$

Now for each $x \in H$, the Fréchet derivative $\phi^{\prime}(x)$ of $\phi$ at $x$ is a member of the space of all continuous linear functionals on $H$ and so it follows that there is a function $\nabla \phi$ on $H$ so that

$$
\phi^{\prime}(x) h=\langle h,(\nabla \phi)(x)\rangle_{H}, x, h \in H .
$$

The function $\nabla \phi$ is called the gradient of $\phi$, the Sobolev gradient of $\phi$ if $H$ is a Sobolev space. What possibilities are there of finding critical points of $\phi$ in a constructive way? 'Constructive' is emphasized since a numerical setting is sought together with a theoretical one. Some results on steepest descent follow.

Theorem 3. Suppose $\phi$ is bounded from below and $\nabla \phi$ is locally lipschitzian. If $x \in H$ there is a unique function $z:[0, \infty) \rightarrow H$ so that

$$
z(0)=x, z^{\prime}(t)=-(\nabla \phi)(z(t)), t \geq 0 .
$$

Moreover

$$
\int_{0}^{\infty}\|(\nabla \phi)(z)\|^{2}<\infty
$$

Theorem 4. If $u$ is an $\omega$-limit point of $z$ as in $(2)$, then $(\nabla \phi)(u)=0$.

Defintion. The statement that a $C^{1}$ function $\phi: H \rightarrow R$ satisfies a gradient inequality (Lojasiewicz inequality) on a region $\Omega \subset H$ means that there is $c>$ $0, \theta \in(0,1)$ so that

$$
\|(\nabla \phi)(v)\|_{H} \geq c \phi(v)^{\theta}, v \in \Omega
$$

Theorem 5. Under the above conditions on $\phi$ if (3) holds and $z$ satisfies

$$
z^{\prime}(t)=-(\nabla \phi)(z(t)), z(t) \subset \Omega, t \geq 0,
$$

then

$$
u=\lim _{t \rightarrow \infty} z(t) \text { exists and }(\nabla \phi)(u)=0 .
$$

In [7] it is shown that (3) holds in finite dimensional cases in a neighborhood of a zero of $\phi$ provided $\phi$ is analytic. A direct generalization to infinite dimensions does not hold (take $T \in L(H, K)$ to be compact and self-adjoint, $H, K$ infinite dimensional Hilbert spaces and define $\left.\phi(x)=\|T x\|_{K}^{2} / 2, x \in H\right)$. In [16], this inequality was extended to some infinite dimensional cases to study asymptotic limits of solutions to time-dependent PDE (see also [2],[5]). Theorem 8 gives a sufficient condition for a gradient inequality to hold.

Theorem 6. Suppose that $G$ is a $C^{1}$ function on $H$ and

$$
\phi(x)=\|x\|_{H}^{2} / 2+G(x), x \in H .
$$

Suppose also that $\phi$ is coercive $\left(\phi(x) \rightarrow \infty\right.$ as $\left.\|x\|_{H} \rightarrow \infty\right), \nabla(G)$ is compact (if $\left\{x_{k}\right\}_{k=1}^{\infty}$ is a bounded sequence in $H$, then $\left\{\nabla G\left(x_{k}\right)\right\}_{k=1}^{\infty}$ has a convergent subsequence) and locally lipschitzian. If $z:[0, \infty) \rightarrow H$ satisfies (2) then $z$ has an $\omega$-limit point and each such point is a zero of $\nabla \phi$. 
For the next theorem, suppose that $K$ is a second Hilbert space, $F: H \rightarrow K$ is $C^{1}$

$$
\phi(x)=\|F(x)\|_{K}^{2} / 2, x \in H,
$$

and $\phi$ has a locally lipschitzian derivative. Note that

$$
(\nabla \phi)(x)=F^{\prime}(x)^{*} F(x), x \in H
$$

where for $x \in H, F^{\prime}(x)^{*}: K \rightarrow H$ is the Hilbert space adjoint of $F^{\prime}(x)$. The next section contains a discussion of adjoints as in (6).

Theorem 7. In addition to (5), suppose that

$$
\|(\nabla \phi)(v)\|_{H} \geq c\|F(v)\|_{K}, v \in B_{r}(x)
$$

(i.e., $\phi$ satisfies (3) with $\theta=\frac{1}{2}$ ). If

$$
\|F(x)\|_{K} \leq r c,
$$

then there is $u \in B_{r}(0)$ so that

$$
F(u)=0
$$

Equation (2) is an ordinary differential equation in infinite dimensions. It serves two roles. The first is numerical in that solutions to (2) can be tracked numerically to obtain approximations to solutions $u$ to $F(u)=0$. This gives the prospect of a unified numerical approach to a very large collection of problems in PDE. In Section 9 one substantial application, out of many, is indicated. The second possibility for (2) is for existence results. For problems in the form (5), existence of a solution $u$ to $F(u)=0$ can be established if a gradient inequality can be shown to hold on a region containing a trajectory $z$ of (2). As indicated in Section 8, the establishment of a gradient inequality is equivalent to the uniform boundedness of solutions to a certain collection of linear problems. A great deal of work remains for problems of this nature.

\section{A Very Quick Look at Sobolev Spaces}

This section is for some who might not make their living with Sobolev spaces. Our general reference for these spaces is [1]. Let's see how one of the simplest Sobolev spaces, $H^{1,2}([0,1])$, may be defined. The elements of $H^{1,2}([0,1])$ are the set of all first terms of the closure $Q$ in $L_{2}([0,1])^{2}$ of

$$
\left\{\left(\begin{array}{c}
u \\
u^{\prime}
\end{array}\right): u \in C^{1}([0,1])\right\}
$$

For $\left(\begin{array}{l}f \\ g\end{array}\right) \in Q, g$ is denoted by $f^{\prime}$, thus extending the notion of differentiability in a very convenient way for purposes of differential equations (note that no two members of $Q$ have the same first term). Since $Q$ is a closed subspace of $L_{2}([0,1])^{2}$ there is an orthogonal projection $P$ of $L_{2}([0,1])^{2}$ onto $Q$. To see an important property of this projection, let $H=H^{1,2}([0,1]), K=L_{2}([0,1])$ and define a derivative operator

$$
D: H \rightarrow K, D f=f^{\prime}, f \in H
$$


We calculate the Hilbert space adjoint $D^{*}: K \rightarrow H$ of $D$. Suppose $g \in K$. Then

$$
\begin{aligned}
\langle D f, g\rangle_{K}=\left\langle f^{\prime}, g\right\rangle_{K}=\left\langle\left(\begin{array}{c}
f \\
f^{\prime}
\end{array}\right),\left(\begin{array}{c}
g \\
0
\end{array}\right)\right\rangle_{K^{2}}=\left\langle P\left(\begin{array}{c}
f \\
f^{\prime}
\end{array}\right),\left(\begin{array}{c}
g \\
0
\end{array}\right)\right\rangle_{K^{2}} & \\
& =\left\langle\left(\begin{array}{c}
f \\
f^{\prime}
\end{array}\right), P\left(\begin{array}{l}
g \\
0
\end{array}\right)\right\rangle_{K^{2}}=\left\langle f, \pi P\left(\begin{array}{l}
g \\
0
\end{array}\right)\right\rangle_{H}, f \in H
\end{aligned}
$$

where $\pi\left(\begin{array}{l}r \\ s\end{array}\right)=r, r, s \in K$. Hence,

$$
\left.D^{*} g=\pi P\left(\begin{array}{l}
g \\
0
\end{array}\right)\right\rangle_{H}
$$

By contrast, if $D$ is considered to be a closed densely defined linear transformation on $K$ (with domain precisely those elements of $K$ which are also in $H$ ), then

$$
D^{t} g=-D g, \text { for all } g \text { such that } g \in H, g(0)=0=g(1),
$$

the conventional adjoint of the derivative operator. Thus we are faced with two different adjoints for the same transformation. Being clear about such occurences is helpful in dealing with Sobolev gradients.

To get an expression for $P$, and hence for $D^{*}$, note that the orthogonal complement in $L_{2}([0,1])^{2}$ of $Q$ is

$$
\left\{\left(\begin{array}{c}
v^{\prime} \\
v
\end{array}\right): v \in H^{1,2}([0,1]), v(0)=0=v(1)\right\}
$$

and so if $\left(\begin{array}{l}f \\ g\end{array}\right) \in L_{2}([0,1])^{2}$, then this element is uniquely the sum of a member of $Q$ and a member of the orthogonal complement of $Q$. The expression of this fact gives a two point boundary value problem for a constant coefficient system of two linear equations. It is an exercise to find an explicit solution to this system.

More generally, if each of $H$ and $K$ is a Hilbert space and $T$ is a closed densely defined linear transformation from $H$ to $K$, a formula of Von Neumann, [19], gives that the orthogonal projection of $H \times K$ onto $\left\{\left(\begin{array}{c}x \\ T x\end{array}\right), x \in D(T)\right\}$ is

$$
\left(\begin{array}{cc}
\left(I+T^{t} T\right)^{-1} & T^{t}\left(I+T T^{t}\right)^{-1} \\
T\left(I+T^{t} T\right)^{-1} & I-\left(I+T T^{t}\right)^{-1}
\end{array}\right) .
$$

In the above, $T^{t}$ represents the adjoint of $T$ as a closed densely defined linear transformation on $H$ to $K$ :

$$
\langle T x, y\rangle)_{K}=\left\langle x, T^{t} y\right\rangle_{H}
$$

for all $x$ in the domain of $T, y$ in the domain of $T^{t}$ (c.f. [15]). Note in passing that $\left(I+T^{t} T\right)^{-1}$ is the embedding operator for $H$ into the space whose points are the domain of $T$ and whose norm is the graph norm of $T$ :

$$
\left(\|x\|_{H}^{2}+\|T x\|^{2}\right)^{\frac{1}{2}}, x \text { in the domain of } T .
$$

For $H$ an $L_{2}$ space of vector valued functions defined on a region $\Omega \subset R^{k}$ and $T$ a transformation on a dense subset of $H$ which, for $u \in H$, yields a list of all partial derivatives of $u$ up to a certain order $m$, the domain of $T$ becomes the Sobolev space $H^{m, 2}(\Omega)$. The norm of each member $u$ of this space is the graph norm of $u$ under $T$. 
Such orthogonal projections are fundamental to the construction of Sobolev gradients in both function space and corresponding finite dimensional approximations. The self-dual nature of such spaces is systematically used despite the emotional attachment of many to the idea that such self-duality is useless (or worse) in the study differential equations. A novella could be written on this topic.

\section{Differential Equations: More Concrete Developments}

We give two ways in which PDEs can be recast as the problem of finding critical points of a function $\phi$, i.e. zeros of $\nabla \phi$. A very simple example illustrates the first way. Experience has shown that someone who codes successfully this example is prepared to code much more complicated problems, problems of scientific interest.

Example. Find $u$ in the Sobolev space $H=H^{1,2}([0,1])$ so that

$$
u^{\prime}-u=0 \text {. }
$$

Define

$$
\phi(u)=\frac{1}{2} \int_{0}^{1}\left(u^{\prime}-u\right)^{2}, u \in H
$$

Something that occurs for many systems of differential equations also happens in this case: a zero of the corresponding Sobolev gradient $\nabla \phi$ is also a zero of $\phi$. Here is a representation of $\nabla \phi$ in the present case.

Define $F: H \rightarrow K=L_{2}([0,1])$ by

$$
F(u)=u^{\prime}-u, \phi(u)=\|F(u)\|_{K}^{2} / 2, u \in H .
$$

Note that

$$
\phi^{\prime}(u) h=\left\langle h^{\prime}-h, u^{\prime}-u\right\rangle_{K}=\left\langle\left(\begin{array}{c}
h \\
h^{\prime}
\end{array}\right),\left(\begin{array}{c}
u-u^{\prime} \\
u^{\prime}-u
\end{array}\right)\right\rangle_{K \times K}, u, h \in H
$$

Denote by $P$ the orthogonal projection onto $Q$ in (9) and define $\pi: K \times K \rightarrow K$ by $\pi\left(\begin{array}{l}f \\ g\end{array}\right)=f, f, g \in K$. Then from $(14)$

$$
\phi^{\prime}(u) h=\left\langle\left(\begin{array}{c}
h \\
h^{\prime}
\end{array}\right), P\left(\begin{array}{c}
u-u^{\prime} \\
u^{\prime}-u
\end{array}\right)\right\rangle_{K \times K}
$$

and so

$$
\phi^{\prime}(u) h=\left\langle h, \pi P\left(\begin{array}{c}
u-u^{\prime} \\
u^{\prime}-u
\end{array}\right)\right\rangle_{H}, h, u \in H
$$

and consequently

$$
(\nabla \phi)(u)=\pi P\left(\begin{array}{c}
u-u^{\prime} \\
u^{\prime}-u
\end{array}\right), u \in H .
$$

This expression for this gradient can be further simplified using an expression for $P$ in the previous section.

A finite dimensional counterpart, to follow, points to a way to code such problems. We remain with the above example but the considerations generalize rather easily to much more complicated problems. 
Pick a positive integer $n$ and divide the interval $[0,1]$ into $n$ pieces of equal length. Take $\delta=\frac{1}{n}$ and define $D_{0}, D_{1}: R^{n+1} \rightarrow R^{n}$ so that if $u=\left(u_{0}, u_{1}, \ldots, u_{n}\right) \in R^{n+1}$ then

and take

$$
\begin{aligned}
& D_{0} u=\left(\frac{u_{1}+u_{0}}{2}, \ldots, \frac{u_{n}+u_{n-1}}{2}\right), \\
& D_{1} u=\left(\frac{u_{1}-u_{0}}{\delta}, \ldots, \frac{u_{n}-u_{n-1}}{\delta}\right)
\end{aligned}
$$

$$
D u=\left(\begin{array}{l}
D_{0} u \\
D_{1} u
\end{array}\right) .
$$

Define a second inner product

$$
\langle u, v\rangle_{S, n}=\langle D u, D v\rangle_{R^{2 n}}, u, v \in R^{n+1} .
$$

A finite dimensional version $\phi_{n}$ of (12) is given by

$$
\phi_{n}(u)=\left\|D_{1} u-D_{0} u\right\|_{R^{n}}^{2} / 2, u \in R^{n+1} .
$$

Note that for $h \in R^{n+1}$

$$
\phi_{n}^{\prime}(u) h=\left\langle D_{1} h-D_{0} h, D_{1} u-D_{0} u\right\rangle_{R^{n}}=\left\langle h,\left(D_{1}-D_{0}\right)^{t}\left(D_{1}-D_{0}\right) u\right\rangle_{R^{n+1}} .
$$

so that

$$
\left(\nabla_{n} \phi_{n}\right)(u)=\left(D_{1}-D_{0}\right)^{t}\left(D_{1}-D_{0}\right) u, u \in R^{n+1},
$$

the ordinary gradient of $\phi_{n}$, that is to say, the list of relevant partial derivatives.

Now for $u \in R^{n+1}, \phi_{n}^{\prime}(u)$ is also a linear functional on $R^{n+1}$ under the norm $\|\cdot\|_{S, n}$. Accordingly there is the function $\nabla_{S, n} \phi_{n}$ so that

$$
\begin{aligned}
\left(\phi_{n}^{\prime}(u) h=\left\langle h,\left(\nabla_{S, n} \phi_{n}\right)(u)\right\rangle_{S, n},=\left\langle D h, D\left(\nabla_{S, n} \phi_{n}\right)(u)\right\rangle_{S, n}\right. & \\
& =\left\langle h, D^{t} D\left(\nabla_{S, n} \phi_{n}\right)(u)\right\rangle_{S, n}, u, h \in R^{n+1} .
\end{aligned}
$$

Thus,

and so

$$
\left(D^{t} D\right)\left(\nabla_{S, n} \phi_{n}\right)(u)=\left(\nabla_{n} \phi_{n}\right)(u)
$$

$$
\left(\nabla_{S, n} \phi_{n}\right)(u)=\left(D^{t} D\right)^{-1}\left(\nabla_{n} \phi_{n}\right)(u), u \in R^{n+1} .
$$

This relationship between ordinary numerical gradient and its Sobolev gradient counterpart is a general phenomenom. A numerical analyst recognizes this Sobolev gradient to be a preconditioned version of the ordinary one. In [4], [11] this statement is reinforced in detail to indicate that finite dimensional Sobolev gradient theory gives an organized approached to preconditioning.

This is a place to point out that if in (12) we sought a gradient $g$ in $L_{2}([0]$,$) by$ which to represent $\phi^{\prime}$ :

$$
\left(\phi^{\prime}(u)\right) h=\langle h, g(u)\rangle_{L_{2}([0,1])}, u, h \in L_{2}([0,1])
$$

the gradient $g$ would be only densely defined on $L_{2}([0,1])$ and discontinuous everywhere it is defined. Such an object is not a promising one for which numerical approximations are to be sought. However, the ordinary gradient of $\phi_{n}$ is just what one would try to use for such an approximation. Now is legendary that such ordinary gradients, defined for problems in differential equations, perform very poorly numerically, the performance degenerating dramatically as the number of mesh points increases. By contrast, the Sobolev gradient of $\phi$ is defined everywhere and is a differentiable function. The Sobolev gradient of $\phi_{n}$ performs very nicely numerically, typically the number of interations required does not depend on the number 
of mesh points chosen. This is an instance of this writer's First Law of Numerical Analysis:

\section{NUMERICAL DIFFICULTIES AND ANALYTICAL DIFFICULTIES ALWAYS COME IN PAIRS}

An orthogonal projection related to the above example is given by

$$
P=D\left(D^{t} D\right)^{-1} D^{t} .
$$

It is the orthogonal projection of $R^{2 n}$ onto the range of $D$. It is an exercise to reconcile this expression with (11).

The above is an indication how a variational principle can be established for many systems of differential equations. If a given system corresponds to the EulerLagrange functional on a Sobolev space, there is another, direct method available. Examples include energy functionals for Hamiltonians, elasticity, transonic flow, Ginzburg-Landau functionals for superconductivity, oil-water separation problems, minimal surfaces, Yang-Mills functionals. Generally, if an energy functional is available, it is better to look directly for critical points of the functional rather than forming a new functional based on the corresponding Euler-Lagrange equations (for one thing, Euler-Lagrange equations are of degree twice that of the maximum order of derivative appearing in the energy functional).

To illustrate this suppose that $n$ is a positive integer and $\Omega$ is a domain in $R^{n}$. Define $\phi$ so that

$$
\phi(u)=\int_{\Omega}\left(\frac{1}{2}\|\nabla u\|_{R^{m}}^{2}+G(u)\right)
$$

for $u \in H, H$ being an appropriately chosen Sobolev space so that $G \in C^{1}$ and $\nabla \phi$ is locally lipschitzian. Suppose also that $\phi$ is bounded below. Then for $u, h \in H$,

$$
\phi^{\prime}(u) h=\int_{\Omega}\left(\langle\nabla h, \nabla u\rangle_{R^{n}}+G^{\prime}(u) h\right)=\left\langle\left(\begin{array}{c}
h \\
\nabla h
\end{array}\right),\left(\begin{array}{c}
(\nabla G)(u) \\
\nabla u
\end{array}\right)\right\rangle_{H \times K},
$$

$K$ being an appropriate $L_{2}$ space. Denoting by $P$ the orthogonal projection of $H \times K$ onto

(16) one has

$$
\left\{\left(\begin{array}{c}
v \\
\nabla v
\end{array}\right): v \in H\right\}
$$

$$
\phi^{\prime}(u) h=\left\langle h, \pi P\left(\begin{array}{c}
(\nabla G)(u) \\
\nabla u
\end{array}\right)\right\rangle_{H}
$$

where here $\pi$ picks out the first component of $P\left(\begin{array}{c}\nabla G(u) \\ \nabla u\end{array}\right)$. Thus we have for a Sobolev gradient of $\phi$

$$
(\nabla \phi)(u)=\pi P\left(\begin{array}{c}
(\nabla G)(u) \\
\nabla u
\end{array}\right)=u-\pi P\left(\begin{array}{c}
u-\nabla G(u) \\
0
\end{array}\right), u \in H .
$$

On bounded regions with a smooth boundary the transformation

$$
u \rightarrow \pi P\left(\begin{array}{c}
u-(\nabla G)(u) \\
0
\end{array}\right)
$$

(where $\pi\left(\begin{array}{l}f \\ g\end{array}\right)=f$ ) may be seen to be compact by following the development of Section 5 for this particular setting. 
Furthermore we have that $u$ is a critical point of $\phi$ if and only if

$$
(\nabla \phi)(u)=0 .
$$

The Sobolev gradient equation (18) is a substitute for the corresponding EulerLagrange equations together with any 'natural' boundary conditions arising from the required integration by parts used in obtaining these equations from $\phi$. The functional (15) contains only first derivatives but the corresponding Euler-Lagrange equations require second derivatives. Use of equation (18) avoids the old problem, already noted by Hilbert, of introducing more derivatives than the basic problem presupposes.

The Sobolev gradient is a continuous function whose zeros are critical points of $\phi$ which may be sought by means of limits at infinity of a solution $z$ to (2). using continuous steepest descent by means of $z:[0, \infty) \rightarrow H$, Contrast this with the usual non constructive (cf [3],[18]) approach to dealing with (15): Define a minimizing sequence $\left\{u_{k}\right\}_{k=1}^{\infty}$, that is

$$
\lim _{n \rightarrow \infty} \phi\left(u_{k}\right)=\inf _{u \in H} \phi(u) .
$$

Then attempt to extract a convergent subsequence of this sequence whose limit is a minimum of $\phi$ and consequently a solution to the corresponding Euler-Lagrange equations. Continuous steepest descent (2) provides a constructive alternative and so it is not surprising that its finite dimensional versions yield viable numerical methods.

Numerical considerations for directly computing critical points of energy functionals such as (15) are quite similar to those for (5). Sometimes for an energy functional it is helpful to define a second function $J$ :

$$
J(u)=\|(\nabla \phi)(u)\|_{H}^{2}, u \in H
$$

and then use $\nabla J$ in place of $\nabla \phi$ in (2). This is used when there are saddle points for $\phi$ for which numerical computations are unstable. Critical points of $\phi$ become local minima of $J$. An alternative point of view is found in [10] which gives a way to constrain trajectories in seeking numerically a critical point. .

\section{Boundary CONDITIONS}

So far little has been said about how present ideas relate to boundary conditions. We give two rather distinct developments on this topic, both of which contain a guide to numerics. We first point out that traditionally the role of boundary conditions in connection with partial differential equations is to give conditions on boundary values under which a given system has a unique solution. It should be recognized that specifying conditions on a boundary of a region (on which solutions are to be found) is often not known to be adequate for specifying a unique solution. Consider for example a Tricomi equation ( $\mathrm{cf}[8]$ ), a version of which is the following: Find $u$ on $\Omega=[0,1] \times[-1,1]$ so that

$$
u_{1,1}(x, y)+y u_{2,2}(x, y)=0,(x, y) \in \Omega .
$$

It is this writer's understanding that after decades of consideration, it is still not known what boundary conditions might be placed on a prospective solution $u$ to (19) in order that there be one and only one solution. A main problem with (19) is that it is elliptic above the real line and hyperbolic below. For elliptic, parabolic or 
hyperbolic problems, ideas about boundary conditions are quite well understood. A problem is that many systems defy such categorization.

We start with the first of two comments on the 'supplementary condition' problem. We deal with problems in the form (5) although our comments apply equally to those given in variation form like (15).

Suppose each of $H, K, S$ is a Hilbert space, $F$ is a $C^{1}$ function from $H$ to $K, B$ is a $C^{1}$ function from $H$ to $S$.

Problem. Find $u \in H$ so that

$$
F(u)=0, B(u)=0 .
$$

Think of the first equation in (20) as specifying a system of ifferential equations and the second equation as supplying supplementary conditions.

A simple example is taken from Section 6 but with the condition $u(0)=1$ placed on a solution $u$. For this, define

$$
F(u)=u^{\prime}-u, B(u)=u(0)-1, u \in H^{1,2}([0,1]) .
$$

Back now to the general case. Take

$$
\phi(v)=\|F(v)\|_{K}^{2} / 2, v \in H
$$

and for each $v \in H$, define

$$
H_{B, v}=\left\{h \in H: B^{\prime}(v) h=0\right\} .
$$

For each $v \in H$, define $P_{B}(v)$ to be the orthogonal projection of $H$ onto $H_{B, v}$. Then

$$
\begin{aligned}
& \phi \prime(v) h=\left\langle F^{\prime}(v) h, F(v)\right\rangle_{K}=\left\langle h, F^{\prime}(v)^{*} F(v)\right\rangle_{H}= \\
& \quad\left\langle h, P_{B}(v) F^{\prime}(v)^{*} F(v)\right\rangle_{H}, v \in H, h \in H_{B, v}
\end{aligned}
$$

so that the corresponding Sobolev gradient is

$$
\left(\nabla_{B} \phi\right)(v)=P_{B}(v) F^{\prime}(v)^{*} F(v), v \in H .
$$

Suppose $x \in H$ and $B(x)=0$ and assume that both $P_{B}$ and $F^{\prime}(\cdot)^{*} F(\cdot)$ are $C^{1}$. Then there is $z:[0, \infty) \rightarrow H$ so that if

$$
z(0)=x \in H, z^{\prime}(t)=-\left(\nabla_{B} \phi\right)(z(t)), t \geq 0,
$$

then

$$
B(z)^{\prime}(t)=B^{\prime}(z(t)) z^{\prime}(t)=-B^{\prime}(z(t))\left(\nabla_{B} \phi\right)(z(t))=0, t \geq 0
$$

since

$$
\left(\nabla_{B} \phi\right)(z(t)) \in N\left(B^{\prime}(z(t)), t \geq 0 .\right.
$$

Thus continuous steepest descent with this Sobolev gradient, starting at $x$, preserves the supplementary condition satisfied by the initial condition, i.e.

$$
B(z(t))=B(x)=0, t \geq 0
$$

and so $B(u)=0$ if

$$
u=\lim _{t \rightarrow \infty} z(t) .
$$

Consider now a second rather general suggestion about the 'supplementary conditions' problem. For purposes of this suggestion we have not tried for maximum generality but rather to present a certain idea in its simplest form. More on this 
issue can be found in [11] and references contained therein. Suppose $H$ is a Hilbert space, $\phi$ is a nonnegative $C^{2}$ function on $H$ and $\nabla \phi$ is the Sobolev gradient of $\phi$. Then if $x \in H$ there is a unique solution $z$ to

$$
z(0)=x \in H, z^{\prime}(t)=-(\nabla \phi)(z(t)), t \geq 0 .
$$

Suppose that for each such $x \in H$ the corresponding solution $z$ to (23) satisfies

$$
u=\lim _{t \rightarrow \infty} z(t) \text { exists, }
$$

and consequently is a critical point of $\phi$. Call $w, y \in H$ equivalent provided there is $u \in H$ so that if $x$ is either of $w$ and $y$, and $z$ satisfies (23), then (24) holds.

This notion of equivalence leads to a foliation of $H$, two members of $H$ being in the same leaf of the foliation if and only if they are equivalent. This leads to a setting in which each leaf of the foliation contains precisely one critical point of $\phi$. An analytical, topological or algebraic (preferably all three of these) understanding of this foliation tags in a potentially useful way each critical point of $\phi$ with a leaf of the foliation.

How does this look in a linear case? Suppose $T$ is a continuous linear transformation from a Hilbert space $H$ to the Hilbert space $K$. Define $\phi: H \rightarrow K$ so that

Then

$$
\phi(x)=\|T x\|_{K}^{2} / 2, x \in H .
$$

$$
\phi^{\prime}(x) h=\langle T h, T x\rangle_{K}=\left\langle h, T^{*} T x\right\rangle_{H}
$$

so that

$$
(\nabla \phi)(x)=T^{*} T x, x \in H .
$$

Pick $x \in H$ and consider $z$ satisfying (23). Note that not only does

$$
u=\lim _{t \rightarrow \infty} z(t) \text { exist, }
$$

but that $u$ is the nearest zero of $T$ to the initial value $x$. An understanding of the resulting foliation would give a hold on the set of all solutions to (19).

\section{Continuous Newton's Method and Nash-Moser Inverse Function THEOREMS}

In the case of Theorem 7 a sufficient condition for (3) to hold on $\Omega$ is the following from [11]:

Theorem 8. Suppose $\Omega \subset H, F: \Omega \rightarrow K$ is $C^{1}$ and there are $M, b>0$ so that if $g \in K$ and $x \in \Omega$, then there is $h \in H$ so that $\|h\|_{H} \leq M$ and

$$
\left\langle F^{\prime}(x) h, g\right\rangle_{K} \geq b\|g\|_{K} \text {. }
$$

Then for $c=2^{-\frac{1}{2}} b / M$,

$$
\|(\nabla \phi)(u)\| \geq c\left(\phi(x)^{\frac{1}{2}} x \in \Omega .\right.
$$

This is the gradient inequality (3) with $\theta=\frac{1}{2}$. Thus a gradient inequality is implied by sufficiently uniform approximate solutions to members of $g$ by elements $F^{\prime}(u) h$. The finding of solutions or approximate solutions $h$ to the linear equations of the form

$$
F^{\prime}(u) h=g,
$$


where $u, g$ are given, is central to the main point of [9]. A recent result which captures much of the spirit of [9] is the following (a proof is very close to one for a slightly different result in [13]):

Theorem 9. Suppose that each of $H, J, K$ is a Banach space (with $H$ compactly embedded in $J), x_{0} \in H, r>0$ and $F: B_{r, H}\left(x_{0}\right) \rightarrow K$ continuous in the $J$-topology with the property that if $u \in B_{r, H}\left(x_{0}\right)$ then there is $h \in B_{r, H}(0)$ so that

$$
\lim _{t \rightarrow 0+} \frac{1}{t}(F(x+t h)-F(x))=-F\left(x_{0}\right) .
$$

Then there is $u \in B_{r, H}$ so that

$$
F(u)=0
$$

Compare Theorem 7 ('Compactly embedded' above means that every bounded sequence in $H$ has a subsequence which converges in $J$ to an element belonging to $H)$.

Closer to Moser's main result in [9] is

Corollary 1. Suppose $M>0, g \in K$, and $H, J, K, r$ are as in Theorem 9. Suppose also that $G: B_{r, H}(0) \rightarrow K$ so that $G(0)=0, G$ continuous in the $J$-topology, and if $y \in B_{r, H}(0)$ there is $h \in B_{M, H}$ so that

$$
\lim _{t \rightarrow 0+} \frac{1}{t}(G(x+t h)-G(x))=g .
$$

Then if $0 \leq t \leq r / M$ there is $u \in B_{r, H}(0)$ so that

$$
G(u)=t g \text {. }
$$

(Just take $F(x)=G(x)-g, x \in B_{r, H}$ and apply Theorem 9).

\section{Numerical Applications}

Quite a number of numerical implementations have been worked out for Sobolev gradients. As a representative application we indicate how the Ginzburg-Landau development for superconductivity fits into our framework. Work described is that of the present author and his long-time collaborator, R.J. Renka (see [14] and references contained therein). The Ginzburg-Landau functional $\phi$ we use is based on a region $\Omega$, chosen here to be a subset of $R^{2}$ although similar considerations hold in $R^{3}$. Take $H=H^{1,2}(\Omega, C) \times H^{1,2}\left(\Omega, R^{2}\right)$ and $\phi: H \rightarrow R$ defined by

$$
\phi(u, A)=\int_{\Omega}\left(\|\nabla u-i u A\|^{2}+\left\|\nabla \times A-H_{0}\right\|^{2}+\frac{\kappa^{2}}{4}\left(|u|^{2}-1\right)^{2}\right),(u, A) \in H,
$$

where $H_{0}$ is a given $C^{1}$ function on $\Omega$. The complex valued function $u$ is an 'order parameter', meaning here that $|u|^{2}$ indicates density of superconducting electrons. The function $\nabla \times A$ indicates an induced magnetic field while $H_{0}$ indicates an imposed magnetic field.

A Sobolev gradient for $\phi$ is constructed along the lines of the simple example in Section 6. The figures gives some representative results. In each of these figures, the magnitude of the magnetic field $\nabla \times A$ corresponding to a critical point of $(25)$ is depicted. In the first figure, the 'flippers' of the squid-like hole are shorter than corresponding flippers in the second figure. In the first figure, there is a 'free' vortex, one not pinned by a given structure whereas in the second figure there is no 
such free vortex. These pictures illustrate how small changes in a superconducting device may lead to substantially different critical points. These pictures, from a code by Robert Renka, were obtained by Barbara Neuberger as part of a program seeking to aid design of superconductors by means of simulations. For purposes of superconducting electronics, one desires to understand how to place holes or moats which will attract vortices in order to leave substantial parts of the device free of vortices, places in which it is attractive to put superconducting circuits. A recent reference for this program is [14]. Other applications are [17], [11] and references therein. These other applications include transonic flow problems and elasticity problems as well as a variety of Ginzburg-Landau type problems. YangMills (cf [6]) functionals are very close in structure to those of the Ginzburg-Landau functional of superconductivity. It is an interesting research problem to code the Yang-Mills functionals using Sobolev gradients.

A principal interest of this writer in these applications of Sobolev gradients is that they give encouragement to the idea that the above described developments are actually moving in the direction of a central theory of partial differential equations. Sobolev gradients have worked in all known instances in which they have been seriously tried.

\section{Conclusion}

The sketch above seeks to place a broad part of PDE into a variational setting, a setting which includes both numerical and theoretical considerations. A key element is the systematic use of self-duality of Sobolev spaces involved. It is briefly indicated how a wide variety of boundary conditions (more accurately supplementary conditions) may be dealt with in a systematic way.

\section{REFERENCES}

[1] R.A. Adams, Sobolev Spaces, Academic Press, 1978.

[2] R. Chill and M. A. Jendoubi, Convergence to steady states in asymptotically autonomous semilinear evolution equations, Nonlinear Analysis 53 (2003), 1017-1039.

[3] L.C. Evans, Weak Convergence Methods for Nonlinear Partial Differential Equations, CBMS Reg. Conf. Ser., Amer. Math. Soc. 1990

[4] Faragó, I., Karátson, J., Numerical solution of nonlinear elliptic problems via preconditioning operators: Theory and applications. Advances in Computation, Volume 11, NOVA Science Publishers, New York, 2002.

[5] S.-Z. Huang and P. Takač, Convergence in gradient-like systems which are asymptotically autonomous and analytic, Nonlinear Anal. 46 (2001), 675-698.

[6] A. Jaffe and C. Taubes Vortices and Monopoles. Structure of Static Gauge Theories, Progress in Physics 2, 1980.

[7] S. Łojasiewicz, Une propriété topologique des sous-ensembles analytiques rés, Colloques internationaux du C.N.R.S.: Les équations aux dérivées partielles, Editions du C.N.R.S., Paris (1963), 87-89.

[8] D. Lupo and K.R. Payne, On the maximum principle for generalized solutions to the Tricomi problem, Commun. Contemp. Math. 2 (2000), 535-557.

[9] J. Moser, A Rapidly Convergent Iteration Method and Non-Linear Differential Equations, Ann. Scuola Normal Sup. Pisa, 20 (1966), 265-315.

[10] John M. Neuberger, A numerical method for finding sign changing solutions of superlinear Dirichlet problems, Nonlinear World 4 (1997), 73-83.

[11] J.W. Neuberger, Sobolev Gradients and Differential Equations, Springer Lecture Notes in Mathematics 1670, 1997. 


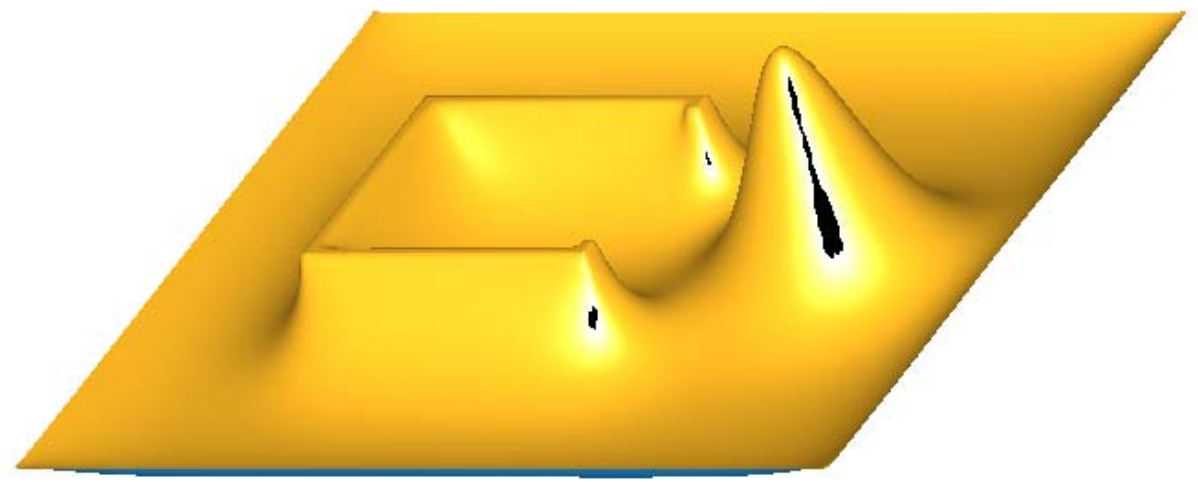

Figure 1. Magnetic Field on a Squid with Smaller Flippers 


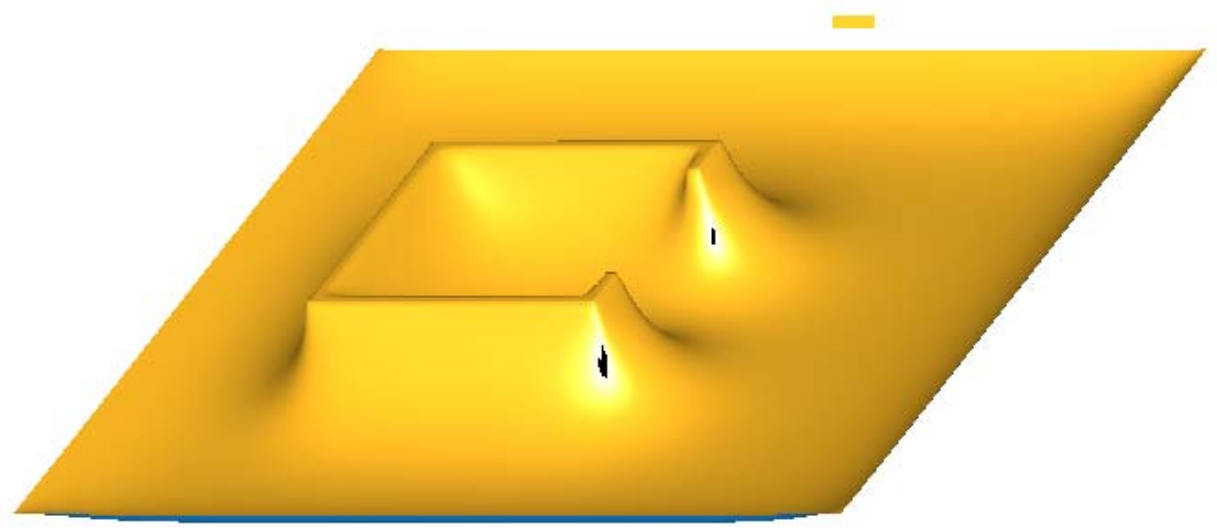

Figure 2. Magnetic Field on a Squid with Longer Flippers 
[12] J.W. Neuberger, Continuous Newton's Method for Polynomials, Mathematical Intelligencer, 21 (1999), 18-23.

[13] J.W. Neuberger, A near minimal hypothesis Nash-Moser Theorem, Int. J. Pure. Appl. Math.. 4 (2003), 269-280.

[14] J. W. Neuberger and R. J. Renka, Numerical determination of vortices in superconductors: simulation of cooling, Supercond. Sci. Technol 16 (2003), 1-4.

[15] F. Reisz and B.-Z. Nagy Functional Analysis, Ungar, 1955.

[16] L. Simon, Asymptotics for a class of non-linear evolution equations with applications to geometric problems, Ann. Math. 118 (1983), 525-571.

[17] S. Sial, J. Neuberger, T. Lookman, A. Saxena, Energy minimization using Sobolev gradients: applications to phase separation and ordering., J. Comp. Physics 189 (2003),88-97.

[18] M. Struwe, Variational Methods, Ergebnisse Mathematik Grenz., Springer, 1996.

[19] J. von Neumann, Functional Operators II, Annls. Math. Stud. 22, (1940).

[20] E. Zeidler, Nonlinear Functional Analysis and its Applications III, Springer-Verlag, 1985.

E-mail address: jwn@unt.edu

Department of Mathematics, University of North Texas, Denton, TX 76203-1430 